\title{
On the nodal structure of single-particle approximation based atomic wave functions
}

\author{
Dario Bressanini ${ }^{\text {a) }}$ and Gabriele Morosi ${ }^{\text {b) }}$ \\ Dipartimento di Scienze Chimiche ed Ambientali, Università dell'Insubria, via Lucini 3, 22100 Como, Italy
}

(Received 27 May 2008; accepted 1 July 2008; published online 5 August 2008)

\begin{abstract}
The nodal structures of atomic wave functions based on a product of spatial orbitals, namely, restricted, unrestricted, and generalized valence bond wave functions, are shown to be equivalent. This result is verified by fixed node-diffusion Monte Carlo simulations for atoms up to Ne. Also for a molecular system, $\mathrm{Li}_{2}$ at the equilibrium geometry, a multideterminantal generalized valence bond wave function does not improve the nodal surfaces of a restricted Hartree-Fock wave function. (C) 2008 American Institute of Physics. [DOI: 10.1063/1.2963501]
\end{abstract}

\section{INTRODUCTION}

The properties of the nodal structure of atomic and molecular wave functions are at the heart of the fixed nodediffusion Monte Carlo (FN-DMC) method. ${ }^{1}$ They directly influence the quality of the results of the simulations, but despite their fundamental importance little is known. Only recently a few papers ${ }^{2-6}$ have begun to study the nodal properties of both exact and approximate wave functions.

In this paper we want to investigate and compare the nodal structure of a family of wave functions written as a product of optimized spatial orbitals times different spin functions. The motivations were that, as we will discuss in the following section, not all these wave functions can satisfy the asymptotic properties the exact wave function is known to possess, and we wanted to investigate how this could affect the nodal structure.

\section{NODAL STRUCTURE}

Among the wave functions based on the approximation that each electron is described by a single-particle function, namely, an orbital, the restricted Hartree-Fock (RHF) wave function, even for a closed shell system, is not the most general one. The orbital double occupancy is removed in the unrestricted Hartree-Fock (UHF) wave function, but this is not an eigenfunction of $S^{2}$, a problem that can be solved within the spin-extended HF method ${ }^{7}$ by projecting a spin eigenfunction. The most general wave function is the spinoptimized self-consistent field (SO-SCF) wave function, ${ }^{8}$ where both spatial orbitals and spin eigenfunctions are optimized. A simplified form is the generalized valence bond (GVB) method, ${ }^{9}$ which optimizes the orbitals for an arbitrary, specified spin coupling.

The conventional wisdom, although not mathematically proven, is that in variational calculations the energies of these wave functions improve according to

\footnotetext{
a)Electronic mail: dario.bressanini@uninsubria.it.

${ }^{b)}$ Electronic mail: gabriele.morosi@uninsubria.it.
}

$$
E\left(\Psi_{\mathrm{RHF}}\right) \geqslant E\left(\Psi_{\mathrm{UHF}}\right)>E\left(\Psi_{\mathrm{GVB}}\right)>E\left(\Psi_{\text {SO-SCF}}\right) .
$$

This ordering is what one should expect considering that the RHF function has less variational freedom, while the UHF function for closed shell atoms is usually similar, if not equal, to the RHF. The GVB function, for both closed and open shell systems, generally gives better energies putting each electron in its own orbital and, differently from the UHF function, still remaining a pure spin state. The SO-SCF wave function improves only slightly upon the previous one as usually just one spin coupling is relevant.

Even more relevant, it is known that the RHF wave function does not satisfy the correct asymptotic behavior when one electron is at large distance.

In general, for an $N$-electron system Patil ${ }^{10}$ has shown that

$$
\begin{aligned}
\phi_{0}^{(N)} \stackrel{r_{1} \rightarrow \infty}{\rightarrow} & r_{1}^{a_{1}}\left[1+c_{1} r_{1}^{-1}+O\left(r_{1}^{-2}\right)\right] \exp \left(-r_{1} / b_{1}\right) Y_{l_{1}}^{m_{1}}\left(\hat{\mathbf{r}}_{1}\right) \phi_{0}^{(N-1)} \\
& \times(2, \ldots, N) .
\end{aligned}
$$

Recursively, setting the right symmetry, we obtain the structure

$$
\Psi=\hat{A}\left(f_{1}(1) f_{2}(2) \ldots f_{N}(N) \Theta^{N}\right),
$$

where $\Theta^{N}$ is the correct $S^{2}$ spin eigenfunction. Each electron has its own orbital, so it is clear that the simple RHF wave function has the wrong behavior, even for the case $N=2$. Note that, in general, the computational cost to evaluate such a function scales exponentially due to the presence of the antisymmetrizer operator.

In DMC, within the FN approximation, the energy depends only on the nodes of the wave function, used as boundary condition for the diffusion process. Two distinct wave functions having the same nodes give exactly the same FN-DMC energy.

It is tempting to argue that, if the quality of the wave function improves, measured by its variational energy, the quality of its nodes should improve, leading to better DMC energies. However, scattered results in the literature have shown that this is not always the case, but to our knowledge a systematic study has never been performed. 
Consider the RHF and UHF wave functions for the nitrogen atom,

$$
\begin{aligned}
& \Psi_{\mathrm{RHF}}=\left|1 s_{R} 2 s_{R} 2 p^{3}\right|\left|1 s_{R} 2 s_{R}\right|, \\
& \Psi_{\mathrm{UHF}}=\left|1 s_{U} 2 s_{U} 2 p^{3}\right|\left|1 s_{U}^{\prime} 2 s_{U}^{\prime}\right|,
\end{aligned}
$$

where the subscripts $U$ and $R$ are meant as a reminder that the orbitals, for the restricted and unrestricted cases, are different. Since the $p$ orbitals are irrelevant in the following discussion we omit the subscript.

If we multiply these functions by a Jastrow factor, we can recover some correlation energy. Their variational energies must be evaluated using the variational Monte Carlo (VMC) algorithm, ${ }^{1}$ and it is expected that the energy ordering of the two functions does not change,

$$
\begin{aligned}
& E_{\mathrm{VMC}}\left(\Psi_{\mathrm{RHF}}\right)>E_{\mathrm{VMC}}\left(\Psi_{\mathrm{UHF}}\right), \\
& E_{\mathrm{VMC}}\left(\Psi_{\mathrm{RHF}} J\right)>E_{\mathrm{VMC}}\left(\Psi_{\mathrm{UHF}} J\right) .
\end{aligned}
$$

If the nodes of the two wave functions are different, it is not possible to say, a priori, which function gives a better DMC energy. Note that the nodes are unchanged by the multiplication of an overall positive Jastrow factor,

$$
E_{\mathrm{DMC}}\left(\Psi_{\mathrm{UHF}} J\right)=E_{\mathrm{DMC}}\left(\Psi_{\mathrm{UHF}}\right) .
$$

Now, we extend the discussion on nodal surfaces of RHF and UHF wave functions by Reynolds et al. ${ }^{11}$ to more general wave functions based on an orbital product. Let us suppose that the UHF wave function gives a better FN energy than the RHF wave function. We can show that, for any given UHF wave function for the $\mathrm{N}$ atom, we can always find a RHF one with the same nodes and hence with the same DMC energy. We can do this by noting two facts: first the nodal topology of the UHF and RHF functions is the same: there are two independent nodes coming from the alpha and beta determinants and intersecting in a lower dimensional submanifold. Second, the node of the beta determinant, built with only $1 s$ and $2 s$ orbitals, is known analytically and is $r_{6}=r_{7}$, assuming we label the first five electrons as alpha, and the last two as beta, something we can always do as long as the Hamiltonian is spin independent. Furthermore, this node does not depend on the exact form of the $1 s$ and $2 s$ orbitals, but only on their spherical symmetry. This means that we can build a restricted wave function, doubly occupying the $1 s_{U}$ and $2 s_{U}$ orbital, having exactly the same nodes of the original unrestricted function,

$$
\begin{aligned}
\Psi_{\mathrm{UHF}} & =\left|1 s_{U} 2 s_{U} 2 p^{3}\right|\left|1 s_{U}^{\prime} 2 s_{U}^{\prime}\right| \rightarrow\left|1 s_{U} 2 s_{U} 2 p^{3}\right|\left|1 s_{U} 2 s_{U}\right| \\
& =\Psi_{\mathrm{RHF}}^{\prime} .
\end{aligned}
$$

Hence

$$
E_{\mathrm{DMC}}\left(\Psi_{\mathrm{RHF}}^{\prime}\right)=E_{\mathrm{DMC}}\left(\Psi_{\mathrm{UHF}}\right) \text {. }
$$

In other words, in the $\Psi_{\mathrm{RHF}}^{\prime}$ function we build the beta determinant with the same orbitals used in the construction of the alpha determinant. Of course the $\Psi_{\mathrm{RHF}}^{\prime}$ function is not the best, from the variational point of view, RHF wave function.
The argument can be reversed: we can also conclude that for any given RHF function, there is always an UHF one (actually an infinite number) with exactly the same nodal structure, and hence giving exactly the same quantum Monte Carlo (QMC) energy.

From this argument it seems not worth to use UHF wave functions in DMC, despite the fact that it has more variational freedom, provided one is able to optimize the FN DMC energy of the more commonly used functions with doubly occupied orbitals. In other words, the RHF wave function with the best DMC energy (not necessarily the one with the best variational energy) has the same DMC energy of the best UHF wave function.

The UHF wave function is still, like the RHF one, a single determinant function. In the more general product of single-particle functions times a single $S^{2}$ spin eigenfunction, the GVB approach, the wave function is expressed as a sum of different determinants, and this could have, in principle, a big effect on the nodal structure, leading to more accurate QMC energies. We have previously seen that a wave function with doubly occupied orbitals cannot satisfy all the boundary conditions that the exact wave function must satisfy, unlike a GVB function, ${ }^{10,12}$ and in principle this could affect the quality of the nodes.

However, we will show that, at least for atoms, this is not the general case.

Consider the structure of the GVB wave function for the $\mathrm{N}$ atom,

$$
\begin{aligned}
\Psi_{\mathrm{GVB}}= & \left|1 s 2 s 2 p^{3}\right|\left|1 s^{\prime} 2 s^{\prime}\right|-\left|1 s^{\prime} 2 s 2 p^{3}\right|\left|1 s 2 s^{\prime}\right| \\
& +\left|1 s^{\prime} 2 s^{\prime} 2 p^{3}\right||1 s 2 s|-\left|1 s 2 s^{\prime} 2 p^{3}\right|\left|1 s^{\prime} 2 s\right| .
\end{aligned}
$$

It is clear that all beta determinants, even if built with different orbitals, have exactly the same node $r_{6}=r_{7}$, for the same reason we discussed above. The alpha determinants might have slightly different nodes, since a common outcome of the GVB optimization is that the $1 s$ and $1 s^{\prime}$ and $2 s$ and $2 s^{\prime}$ orbitals differ little. So the nodal topology of this function is similar to the one of the monodeterminantal functions, with two intersecting nodal surfaces, generating a minimum of four nodal regions. One surface is $r_{6}=r_{7}$, while the second surface is the result of the weighted combination of the nodes of the alpha determinants. It has been conjectured (and numerically verified in some cases) that the exact ground state of an atomic and molecular system has exactly two nodal regions. ${ }^{13,14}$ For this reason, using a GVB function, although it is able to satisfy the asymptotic boundary conditions, does not seem to improve the nodal structure. A small, well chosen, configuration interaction (CI) or multiconfiguration self-consistent field expansion can have a much more dramatic effect in modifying the nodes of the single determinant wave function. ${ }^{2}$

The above arguments can be rigorously applied only to atoms up to $Z=7$. As soon as the beta determinant has electrons in $p$ orbitals, the nodal structure becomes too difficult to treat algebraically. However, we will show numerically that even up to $Z=10$ a GVB wave function does not improve the nodal structure of a single determinant wave function, measured by the FN-DMC energy. 
TABLE I. Energies (hartree) and correlation energy percentages.

\begin{tabular}{lccccccccc}
\hline \hline & $\mathrm{HF}^{\mathrm{a}}$ & $\mathrm{NRL}^{\mathrm{a}}$ & $\mathrm{VMC} \mathrm{HF}$ & \%CE & VMC GVB & \%CE & DMC HF & \%CE & DMC GVB \\
\hline $\mathrm{He}$ & -2.86168 & -2.903724 & $-2.8933(2)$ & 75.3 & $-2.9022(1)$ & 96.5 & $-2.9037(1)$ & 100.0 & $-2.9036(1)$ \\
$\mathrm{Li}$ & -7.432727 & -7.47806 & $-7.4704(1)$ & 83.1 & $-7.4755(1)$ & 94.6 & $-7.4780(1)$ & 99.9 & $-7.4780(1)$ \\
$\mathrm{Be}$ & -14.573023 & -14.66736 & $-14.6261(2)$ & 56.3 & $-14.6312(2)$ & 61.7 & $-14.6575(1)$ & 89.5 & $-14.6570(2)$ \\
$\mathrm{B}$ & -24.529061 & -24.65393 & $-24.5858(3)$ & 45.5 & $-24.5971(2)$ & 54.6 & $-24.6384(1)$ & 87.6 & $-24.6369(1)$ \\
$\mathrm{C}$ & -37.688619 & -37.8450 & $-37.7568(5)$ & 43.6 & $-37.7626(2)$ & 47.3 & $-37.8292(3)$ & 89.9 & $-37.8231(1)$ \\
$\mathrm{N}$ & -54.400934 & -54.5893 & $-54.4620(4)$ & 32.5 & $-54.4780(3)$ & 40.9 & $-54.5732(2)$ & 91.4 & $-54.5746(3)$ \\
$\mathrm{O}$ & -74.809398 & -75.067 & $-74.8708(6)$ & 23.9 & $-74.9233(4)$ & 44.2 & $-75.0501(4)$ & 93.5 & $-75.0501(3)$ \\
$\mathrm{F}$ & -99.409349 & -99.734 & $-99.4685(5)$ & 18.2 & $-99.5567(7)$ & 45.4 & $-99.7150(4)$ & 94.2 & $-99.680(1)$ \\
$\mathrm{Ne}$ & -128.547098 & -128.9383 & $-128.590(1)$ & 11.2 & $-128.7348(7)$ & 48.0 & $-128.9027(3)$ & 90.9 & $-128.8796(6)$ \\
\hline \hline
\end{tabular}

${ }^{\mathrm{a}}$ Hartree-Fock and nonrelativistic limit from Ref. 21.

\section{CALCULATIONS}

We studied the ground state of the first row atoms. In order to investigate the effect of the analytic structure of the wave function and to separate it from the influence of the quality of the basis set, we decided to describe the $1 s, 2 s$, and $2 p$ orbitals in a very compact way.

$$
\begin{aligned}
& 1 s=e^{\left(a r+b r^{2}\right) /(1+r)}, \quad 2 s=(r-c) e^{\left(a r+b r^{2}\right) /(1+r)}, \\
& 2 p_{x}=x e^{\left(a r+b r^{2}\right) /(1+r)} .
\end{aligned}
$$

In a previous study ${ }^{12}$ we showed that using this kind of functions it is possible to develop very compact, but nevertheless accurate wave functions for two-electron systems. One important property of these functions is that the exponential behavior close to the origin is completely decoupled from the exponential behavior at large distance. If $r \rightarrow 0$ these functions reduce to simple hydrogenoid functions. Each electron is described by a single function. In order to reduce the number of variational parameters, to simplify our analysis, the various $a$ parameters have been fixed to the theoretical hydrogenoid value, i.e., for the $1 s a=-Z$, to fix the cusp conditions, while for the $2 s$ and $2 p$ orbitals $a=-Z / 2$. When we tried to optimize these parameters, very little gain was observed at the VMC level and nothing at the DMC level. The parameter $c$ of the $2 s$ orbital, on the other hand, was found to directly influence the FN-DMC energy and was left free to be optimized, together with the $b$ parameters.

Even if the nodal structure is not directly influenced by the correlation factor, we used a simple Jastrow factor, in order to reduce the variance of the estimated energy. We optimized the wave functions by minimizing the variance of the local energy using VMC simulations. ${ }^{15}$ The RHF determinants have a maximum of five optimizable parameters: one each for the Jastrow, the $1 s$, and the $2 p$ orbitals, and two for the $2 s$ orbitals. For the GVB wave functions the number of orbital parameters is doubled. Due to the compact form we chose for the orbitals, there are no linear parameters coming from linear combination of basis functions. In all cases the optimized parameters, when plotted against the atomic number, show a linear behavior. We exploited this empirical fact to provide some good starting values of the parameters and to check if during the nonlinear optimization something went wrong. Sometimes the nonlinear optimization could not escape a bad local minimum. By providing a starting point for the parameters coming from an extrapolation between neighboring atoms we could often improve the energy. We performed both VMC and DMC simulations using an average of 5000 walkers.

\section{RESULTS}

The results, both VMC and DMC, are shown in Table I.

In Fig. 1 we plot the correlation energy recovered for the various functions as a function of $Z$. We use this way of displaying the data instead of the more common use of the percentage of correlation energy recovered since we think it gives a different insight, more useful to judge the trend as a function of $Z$. The percentage of the recovered correlation energy can be found in Table I.

The plot shows that the correlation energy to be recovered increases roughly quadratically with $Z$. The closer we are to this exact curve the more accurate are the calculations. Let us first comment on the VMC simulations. The RHF functions recover approximately only a constant amount, entirely due to the two-body Jastrow factor. It is surely possible to use more sophisticated correlation factors, including threebody correlations, but we did not use them since our aim was to study the effect of the form of the wave function on the nodal structure, which is not affected by the correlation factor. The effect of better correlation factors is evidenced by the results by Luchow and Anderson, ${ }^{16}$ also reported in Fig. 1. Their correlation energies, at the VMC level, recovered using three-body Schmidt-Moskowitz correlation factors, ${ }^{17}$

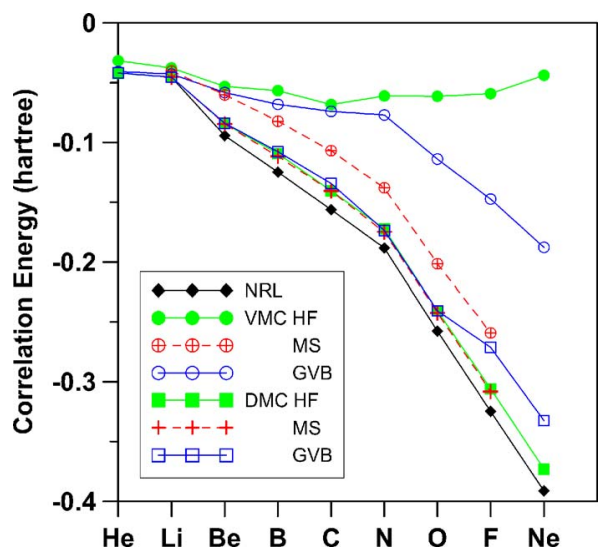

FIG. 1. (Color online) Correlation energy (hartree) for VMC and DMC calculations. MS results are from Ref. 16. 
show the correct quadratic trend. It is interesting to note that starting from the Be atom, the missing correlation energy is approximately constant, and it is tempting to speculate that this is due to the wrong nodal topology of the RHF wave function, having four nodal regions instead of two. For He and $\mathrm{Li}$ the RHF function has a topologically correct nodal structure: for He there are no nodes and for Li only one nodal surface. Although the nodes are not directly relevant when discussing VMC results, if the topology of the nodal structure is wrong, the improvement of the wave function by a positive-everywhere correlation factor is limited, as it cannot move or vary the number of nodes. In other words including higher terms in the correlation factor is not sufficient to converge to the exact value.

Our GVB wave functions, at the VMC level, give better results than the corresponding RHF functions, but only after the nitrogen atom the recovered correlation energy shows a nonconstant trend and a marked improvement over the doubly occupied determinant result.

It is clear that including the correct analytical structure is beneficial in the cases where the $p$ orbitals start to be doubly occupied. The GVB structure gives enough freedom to the $p$ electrons to avoid each other and this has a marked effect: in the $\mathrm{Ne}$ case the correlation energy recovered is almost half of the total one. We stress that this is not due to three-body correlation terms, since the GVB theory is still a one-particle theory and only pair correlation functions were included.

Let us now consider the DMC results. At the DMC level our RHF wave functions recover from $87 \%$ to $100 \%$ of the correlation energy, and again they miss an almost constant amount starting from the Be atom. Although the purpose of this work was not to generate very accurate wave functions, it is interesting to note that this very compact and simple wave function recovers almost the same energy as the more sophisticated HF quality times Schmidt-Moskowitz term used by Luchow and Anderson. ${ }^{16}$ Again, in both cases, the missing correlation energy is due to the wrong nodal structure. Consider now the DMC energy of the GVB functions. Although these functions have the correct analytical asymptotic structure, unlike the simple RHF functions, this fact does not seem to affect the nodal structure. Paradoxically, the fact that the GVB functions have more variationally optimizable parameters, in two cases ( $\mathrm{F}$ and $\mathrm{Ne}$ ) leads to worse results, presumably due to local minima in the variational space, since the parameters have been optimized at the VMC level using the variance minimization algorithm. The GVB functions are more costly to evaluate due to their multideterminantal structure, and more difficult to optimize due to the greater number of parameters, and do not seem to offer any advantage at the DMC level, even if they improve the VMC energy by including the correct asymptotic structure. This is in accordance with our theoretical argument in the previous section. Furthermore, it is worth noting that the difference between the correlation energy recovered with DMC by our simple functions and the one recovered with more sophisticated functions having the same structure ${ }^{16}$ is small, our being usually only slightly less, while the missing correlation energy usually is much greater. In this respect it seems more useful, in general, to direct more computational
TABLE II. Energies (hartree) for $\mathrm{Li}_{2}$ at 5.05 bohrs.

\begin{tabular}{lll}
\hline \hline Wave function & VMC & DMC \\
\hline RHF 1 det. & $-14.9523(2)$ & $-14.9916(1)$ \\
GVB 8 dets. & $-14.9688(1)$ & $-14.9915(1)$ \\
CI 3 dets. & $-14.9632(1)$ & $-14.9931(1)$ \\
GVB 24 dets. & $-14.9782(1)$ & $-14.9936(1)$ \\
CI 3 dets. ${ }^{a}$ & & $-14.9933(2)$ \\
CI 5 dets. & & $-14.9952(1)$ \\
NRL $^{\text {b }}$ & & -14.9954 \\
\hline \hline
\end{tabular}

${ }^{\mathrm{a}}$ Reference 2 .

${ }^{\mathrm{b}}$ Reference 22 .

energies toward wave functions that can recover the missing energy by altering directly the nodal structure. ${ }^{18-20}$ To give a concrete example, with the simple four parameter RHF wave function used in this work, for the nitrogen atom, DMC recovers about $91 \%$ of the correlation energy, to be compared with the 93\% obtained using the Clementi-Roetti wave function, with 27 parameters.

\section{GVB FOR MOLECULES}

Historically, the VB and the GVB approach have been developed with the aim to correctly describe the bond breaking process, and only later applied to atoms. It is known that the RHF wave function incorrectly describes the bond breaking process, even for the simple hydrogen molecule, while the correct dissociation limit is recovered using VB or GVB functions. Mixing covalent and ionic structures, the RHF wave function has a wrong nodal structure asymptotically. It could be speculated that this could somehow introduce some nodal error even at distances close to the equilibrium, since the nodal structure changes smoothly from the dissociation to the equilibrium distance. In order to test this hypothesis we performed some $\mathrm{DMC}$ simulations of the $\mathrm{Li}_{2}$ molecule at the equilibrium distance using a RHF and a GVB wave function, built using as basis set the compact functions previously described, times a simple two-body Jastrow factor. The GVB wave function has eight determinants. As shown in Table II, passing from the RHF to the GVB wave function, the VMC energy improves by 16 mhartree, but the nodal structure seems unchanged since the DMC energy is statistically the same. It was found in previous studies ${ }^{2}$ that adding the determinants $1 \sigma_{g}^{2} 1 \sigma_{u}^{2} 1 \pi_{x}^{2}$ and $1 \sigma_{g}^{2} 1 \sigma_{u}^{2} 1 \pi_{y}^{2}$ to the RHF trial wave function improves the DMC energy. We built a simple CI expansion with three determinants, using the compact basis functions described previously. As expected the DMC energy improves with respect to the GVB one, but interestingly the VMC result is still inferior to the GVB one due to the double occupancy of the orbitals. We removed this constraint using the GVB methodology to the three determinant CI expansion, obtaining an expansion of 24 determinants. The VMC energy improves quite sensibly, but again the DMC energy has a negligible improvement. As comparison we included in Table II the best three determinant wave function we optimized in a previous work on the $\mathrm{Li}_{2}$ system ${ }^{2}$ and the energy obtained by a well chosen five determinants expansion, recovering almost the exact energy. We observe here the same 
phenomenon noted in the case of the atoms: using a GVB trial wave function improves the variational energy, but not the FN energy.

\section{CONCLUSIONS}

The aim of this work was to investigate the effect on the nodal surfaces of changing the analytical structure of the wave functions, restricting them to a product of orbitals, either doubly or singly occupied. From our work it seems that more sophisticated single-particle-based wave functions, beyond the simple RHF, while recovering more energy at the VMC level, do not seem to improve the nodal structure. Simply adding more determinants does not necessarily change the topology of the nodal structure, if those determinants are just coming from a GVB approach. This is in accord with a previous study ${ }^{2}$ that found that only determinants built with different angular momentum couplings than the ground state determinant could improve the nodal structure of the RHF wave function.

\section{ACKNOWLEDGMENTS}

This work was supported by Italian MIUR Grant No. 2006030944.

${ }^{1}$ B. L. Hammond, W. A. Lester, Jr., and P. J. Reynolds, Monte Carlo
Methods in Ab Initio Quantum Chemistry, 1st ed. (World Scientific, Singapore, 1994).

${ }^{2}$ D. Bressanini, G. Morosi, and S. Tarasco, J. Chem. Phys. 123, 204109 (2005).

${ }^{3}$ D. Bressanini and P. J. Reynolds, Phys. Rev. Lett. 95, 110201 (2005).

${ }^{4}$ T. C. Scott, A. Luchow, D. Bressanini, and J. D. Morgan, Phys. Rev. A 75, 060101 (2007)

${ }^{5}$ L. Mitas, Phys. Rev. Lett. 96, 240402 (2006).

${ }^{6}$ M. Bajdich, L. Mitas, G. Drobny, and L. K. Wagner, Phys. Rev. B 72, 075131 (2005).

${ }^{7}$ P.-O. Löwdin, Phys. Rev. 97, 1509 (1955).

${ }^{8}$ U. Kaldor and F. E. Harris, Phys. Rev. 183, 1 (1969).

${ }^{9}$ W. A. Goddard III, Phys. Rev. 157, 73 (1967).

${ }^{10}$ S. H. Patil, J. Phys. B 22, 2051 (1989).

${ }^{11}$ P. J. Reynolds, R. N. Barnett, and W. A. Lester, Jr., Int. J. Quantum Chem., Quantum Chem. Symp. 18, 709 (1984).

${ }^{12}$ D. Bressanini and G. Morosi, J. Phys. B 41, 145001 (2008).

${ }^{13}$ D. M. Ceperley, J. Stat. Phys. 63, 1237 (1991).

${ }^{14}$ W. A. Glauser, W. R. Brown, W. A. Lester, D. Bressanini, B. L. Hammond, and M. L. Koszykowski, J. Chem. Phys. 97, 9200 (1992).

${ }^{15}$ C. J. Umrigar, K. G. Wilson, and J. W. Wilkins, Phys. Rev. Lett. 60, 1719 (1988).

${ }^{16}$ A. Luchow and J. B. Anderson, J. Chem. Phys. 105, 7573 (1996).

${ }^{17}$ K. E. Schmidt and J. W. Moskowitz, J. Chem. Phys. 93, 4172 (1990).

${ }^{18}$ M. Bajdich, L. Mitas, G. Drobny, L. K. Wagner, and K. E. Schmidt, Phys. Rev. Lett. 96, 130201 (2006).

${ }^{19}$ M. D. Brown, J. R. Trail, P. L. Rios, and R. J. Needs, J. Chem. Phys. 126, 224110/1 (2007).

${ }^{20}$ M. Casula and S. Sorella, J. Chem. Phys. 119, 6500 (2003).

${ }^{21}$ E. R. Davidson, S. A. Hagstrom, S. J. Chakravorty, V. M. Umar, and C. F. Fischer, Phys. Rev. A 44, 7071 (1991).

${ }^{22}$ C. Filippi and C. J. Umrigar, J. Chem. Phys. 105, 213 (1996). 